
\title{
28 Resarch Square \\ Citric Acid Coated Iron Oxide Nanoparticles as Contrast Agent for Magnetic Resonance Imaging
}

\author{
F. Y. Alzoubi \\ H. N. Al Jabaly \\ O. A. Abu Noqta \\ H. M. Al-Khateeb \\ M. K. Alqadi \\ Mohamed Bououdina ( $\square$ mboudina@gmail.com ) \\ University of Bahrain https://orcid.org/0000-0001-8770-7129
}

\section{Research Article}

Keywords: Iron oxides, Nanoparticles, Citric acid, Contrast agent, Magnetic resonance imaging

Posted Date: March 7th, 2022

DOI: https://doi.org/10.21203/rs.3.rs-1390032/v1

License: @ (i) This work is licensed under a Creative Commons Attribution 4.0 International License. Read Full License 


\section{Abstract}

Iron oxide nanoparticles (IONPs) were synthesized at varying pH (8 to 12) then subsequently coated with citric acid by co-precipitation as potential magnetic resonance imaging (MRI) contrast agents. The X-ray diffraction patterns confirmed that the structure of the as-synthesized powders is pure nanocrystalline magnetite $\mathrm{Fe}_{3} \mathrm{O}_{4}$ phase. FTIR analysis of citric acid-coated IONPs indicated the presence of peaks at 1357.70 and $1533.97 \mathrm{~cm}^{-1}$, thus confirming the binding of citric acid on the surface of magnetite nanoparticles. Zeta potential measurements indicated that the citric acid-coated IONPs presents much-improved stability compared to bare IONPs; the Zeta potential absolute value is almost three times higher. Finally, citric acid-coated IONPs as a contrast agent for MRI clearly demonstrates their excellent ability to shorten $T_{2}$ and $T_{1}$ relaxation times by more than $94.7 \%$ and $67.9 \%$, respectively. These results reveal that the citric acid-coated IONPs are excellent candidates for potential biomedical application primarily as MRI contrast agents.

\section{Introduction}

Iron oxide nanoparticles (IONPs) attracted great attention because of their outstanding and tunable chemical, optical and magnetic properties that are dependent on their morphology and size, chemical composition, and structural arrangement. These properties make them more suitable particularly for medical and pharmaceutical fields. Their nanometer size facilitates their movement (transport) within the body without disabling normal functions and may reach inaccessible locations. Moreover, IONPs possess additional distinctive characteristics such as availability, biochemical stability, biocompatibility, and the ability to be manufactured in various shapes and sizes [1, 2]. Specifically, IONPs exhibit superparamagnetic behavior and high surface area for biomedical applications, primarily in theragnostic (simultaneously as a contrast agent and magnetic fluid hyperthermia for cancer treatment) and targeted drug delivery. They provide a substantial imaging contrast in magnetic resonance imaging (MRI) by picking up the resonance absorption and consequently the relaxation process is accelerated. In drug delivery, the magnetic properties of IONPs can be manipulated using an external magnetic field so that the therapeutic procedure can be performed at the specified locations/organs [3-5].

Various methods have been adopted for the synthesis of IONPs such as thermal decomposition, coprecipitation, hydrothermal, and reverse micelles. Both thermal decomposition and hydrothermal methods have advantages for the production of unilateral IONPs, besides being flexible and adjustable [6]. It is possible to adopt thermal decomposition of iron five-carbonyl in the presence of oleic acid at $100^{\circ} \mathrm{C}$. Also, it could be oxidized using tri-diethyl to produce highly crystalline and monodispersed maghemite nanoparticles. However, high reaction temperatures limited their applicability to biomedical applications [7]. Besides, such methods are complex and require very high temperatures, compared to the co-precipitation method which is commonly used to produce water-soluble and biocompatible IONPs. The as-prepared IONPs with a suitable surface functionality are effective for biomedical applications [8, 9]. .

Although IONPs exhibit interesting and controllable magnetic properties, they have high tendency to agglomerate as consequence prevent particles' surface functionalization with biocompatible entities such as citric acid. IONPs with sizes in the 1-100 nm can be either ferromagnetic or superparamagnetic (ability to have zero magnetism in the absence of external magnetic field). Superparamagnetic order occurs when the size of 
the magnetic domain is so small that the surrounding thermal energy is sufficient to induce the free rotation of the crystals, known as Brownian motion $[10,11]$.

Extensive research in the literature focused on the preparation and understanding the magnetic behavior of IONPs. However, the reported synthesis routes faced challenges of producing suitable sized, shaped, structured and monodispersed IONPs with high magnetic moments. Although, several review papers have presented indepth and breadth discussion and comparison on the synthesis of IONPs, most focused on the chemical methods. The synthetic routes can be classified into three: physical, chemical and biological methods. The chemical method is the most cited method of producing IONPs [6]. To prevent agglomeration, the assynthesized IONPs are subsequently stabilized via peptization effect or steric stabilization. The use of several peptizing agents such as tetramethyl ammonium hydroxide, perchloric acid, citric acid, nitric acid and acrylic acid have been reported [12].

Several authors reported one pot synthesis process that combines both co-precipitation and stabilization procedure. This process involves additional stabilizing or peptizing agents such as citric acid, oleic acid, or a polymer during the co-precipitation process $[13,14]$. IONPs of $10-100 \mathrm{~nm}$ can be synthesized by controlling the reaction conditions such as temperature, $\mathrm{pH}$, stirring speed, aging time and ionic strength of the medium [1517]. The saturated magnetization of IONPs produced via this method decreases by decreasing the particle size [18].

Sol-Gel method is also known as chemical solution deposition method which involves conversion of the precursor solution into an inorganic solid by chemical means [19]. This approach is an appropriate wet chemical method to synthesize metal oxide nanostructures with specific properties [20].

It is important to highlight that the behavior of IONPs in addition to their large magnetic susceptibility causes microscopic field heterogeneity and activation of proton removal in the presence of an external magnetic field. Therefore, it can be used as MRI probe (contrast agent) to shorten $\mathrm{T}_{2}$ and $\mathrm{T}_{1}$ relaxation times of the neighboring regions [21]. In this study, highly stable and monodispersed IONPs coated with citric acid are synthesized by one-pot co-precipitation method. Particular emphasis is devoted to examining the sensitivity of IONPs as MRI contrast agent in agarose gel phantom.

\section{Materials And Methods}

\subsection{Samples preparation}

$\mathrm{Fe}_{3} \mathrm{O}_{4}$ nanoparticles were synthesized by co-precipitation method of ferrous $\left(\mathrm{Fe}^{+2}\right)$ and ferric $\left(\mathrm{Fe}^{+3}\right) \mathrm{chlorides}^{2}$ with $\mathrm{NaCl}$ and sodium hydroxide $(\mathrm{NaOH})$. $\mathrm{NaCl}$ solution was prepared by adding $11.688 \mathrm{~g}$ of $\mathrm{NaCl}$ to $200 \mathrm{ml}$ of deionized water (DIW). $\mathrm{FeCl}_{2} \cdot 4 \mathrm{H}_{2} \mathrm{O}(0.795 \mathrm{~g})$ and $\mathrm{FeCl}_{3} \cdot 6 \mathrm{H}_{2} \mathrm{O}(1.297 \mathrm{~g})$ were dissolved in $40 \mathrm{ml}$ of DIW under nitrogen gas. Following the complete dissolution of the mixture at room temperature, $25 \mathrm{ml}$ of $1.5 \mathrm{M}$ of sodium hydroxide was added dropwise to the reaction mixture, under continuous vigorous stirring at $500 \mathrm{rpm}$ for 2 hours. Only the $\mathrm{pH}$ value was varied from 8 to 12 by controlling the added amount of sodium hydroxide.

To prevent agglomeration of $\mathrm{Fe}_{3} \mathrm{O}_{4}$ nanoparticles and guarantee a high state of stability, $1.5 \mathrm{~g}$ of citric acid dissolved in $150 \mathrm{ml}$ of DIW was added to the mixture described above for the sample prepared at $\mathrm{pH} 10$, then 
the new mixture was stirred for $15 \mathrm{~min}$. at $70^{\circ} \mathrm{C}$. $\mathrm{Fe}_{3} \mathrm{O}_{4}$ nanoparticles coated with citric acid were collected through a permanent magnet and thoroughly rinsed twice with distilled water to eliminate unreactive or inert impurities.

\subsection{Samples characterizations}

The crystalline structure of the as-prepared IONPs was checked by X-ray diffraction (XRD) using PANalytical Xpert PRO MRD PW 3040 diffractometers equipped with CuKa radiation source $(\lambda=1.5418 \AA)$. The structural bonding and functional groups were determined by Fourier transformed infrared (FTIR) using the Perkin ElmerGX-1FTIR spectrophotometer. Zeta potential measurement was performed using Malvern instrument (Nano ZS model, ZEN 3600). The chemical composition was determined by atomic absorption spectroscopy (Shimadzu AAS-6650).

\subsection{MRI measurements}

For Magnetic Resonance measurements, IONPs synthesized at pH 10 and coated with citric acid was chosen. MRI phantoms were prepared from Agarose gel "Nutrient Agar" by dissolving $1 \mathrm{~g}$ of agarose in $100 \mathrm{ml}$ of distilled water at $80^{\circ} \mathrm{C}$. When fully dissolved, the agarose gel mixture was subsequently simmered down to $50^{\circ} \mathrm{C}$. To prepare optimized IONPs sample, the concentration of $1 \mathrm{mg} / \mathrm{mL}$ was diluted to $(0.5,0.25,0.125$ $\mathrm{mg} / \mathrm{mL}$ ) and then centrifuged. The diluted samples were poured into glass vials containing $3 \mathrm{ml}$ of $1 \mathrm{wt} \%$ Agar gel solution. The solutions were mixed well to achieve homogeneity and then kept cold until hardened. Finally, five agarose gel phantoms containing different concentrations of citric acid coated IONPs (Blank sample 0, $0.125,0.25,0.5$ and $1 \mathrm{mg} / \mathrm{mL}$ ) were prepared for MRI measurements.

\section{Results And Discussion}

\subsection{X-ray diffraction analysis}

XRD patterns of the final powders are shown in Fig. 1. The XRD patterns exhibit well-defined broad and relatively low intensity peaks typical characteristics' of nanocrystalline phases. The peaks located at $2 \theta 30.17^{\circ}$, $35.43^{\circ}, 43.45^{\circ}, 57.07^{\circ}$ and $63.16^{\circ}$ are indexed as (220), (311), (400), (511) and (440) reflections corresponding to the magnetite $\mathrm{Fe}_{3} \mathrm{O}_{4}$ phase with a cubic structure (Fd-3m space group), in agreement with JCPDS card No. 01-075-0449 for magnetite. No additional peaks can be detected hence confirming the purity of the as-prepared nanoparticles irrespective of $\mathrm{pH}$ value, besides it indicates that citric acid coating does not induce any phase transformation of bare $\mathrm{Fe}_{3} \mathrm{O}_{4}[22-24]$.

The average crystallite size ( $\mathrm{D}$ in $\mathrm{nm}$ ) has been determined by means of the well-known Debye-Scherrer's formula given by [25]:

$D=K \lambda / \beta_{h: l} \cos \theta_{1}$ where $\mathrm{K}$ is grain shape constant (0.89 for spherical shape), $\lambda=0.154056 \mathrm{~nm}$ is the wavelength of XRD beam, $\beta_{\mathrm{hkl}}$ is the full width at half maximum for the diffraction peak (radian), and $\theta$ is the diffraction angle. The effect of $\mathrm{pH}$ on the size of IONPs is presented in Table 1. It is clear that the crystallite size increases gradually as the $\mathrm{pH}$ of the solution increases from 8 to 12 . However, at the $\mathrm{pH} 10$, it is interesting to note that crystallite size decrease after citric acid; i.e., 11.91 to $10.19 \mathrm{~nm}$ respectively. According to the obtained results, IONPs prepared 
at pH 10 manifest a dark black color and are more stable compared to the remaining set of IONPs, hence were subsequently coated with it was citric acid and used for MRI measurements.

Table 1

Effect of $\mathrm{pH}$ of solution on the crystallite size of $\mathrm{Fe}_{3} \mathrm{O}_{4}$

nanoparticles.

\begin{tabular}{|ll|}
\hline pH of Solution & Size of $\mathrm{Fe}_{3} \mathrm{O}_{\mathbf{4}}$ Nanoparticles (nm) \\
\hline $\mathbf{8}$ & 10.55 \\
$\mathbf{9}$ & 11.18 \\
$\mathbf{1 0}$ & 11.91 \\
\hline $\mathbf{1 1}$ & 13.44 \\
\hline $\mathbf{1 0}$ (coated) & 10.19 \\
\hline
\end{tabular}

\subsection{FTIR analysis}

The as-prepared IONPs are characterized by FTIR in order to detect functional groups and characterize the existing covalent bonding. Figure 2 presents a typical FTIR spectrum recorded for IONPs prepared at pH 10 and coated with citric acid. The strong absorption band centered at $418.85 \mathrm{~cm}^{-1}$ is assigned to the Fe-O bond, which confirms the formation of $\mathrm{Fe}_{3} \mathrm{O}_{4}$ phase [26]. The peak at $1357.70 \mathrm{~cm}^{-1}$ can be ascribed to the asymmetric stretching of $\mathrm{C}-\mathrm{O}$ from the carboxylic group [27]. Citric acid binds to $\mathrm{Fe}_{3} \mathrm{O}_{4}$ particles' surface through carboxylate group. The $1600 \mathrm{~cm}^{-1}$ peak assigned to the $C=0$ vibration (symmetric stretching) from the $\mathrm{COOH}$ group of citric-acid (CA) shifts to an intense band at about $1533.97 \mathrm{~cm}^{-1}$ for citric acid coated $\mathrm{Fe}_{3} \mathrm{O}_{4}$, hence confirming the binding of a citric acid on the surface of magnetite. A broad and intense band at 3185.70 $\mathrm{cm}^{-1}$ can be assigned to the structural $\mathrm{OH}$ groups as well as to the traces of molecular water and citric acid.

\subsection{Zeta potential analysis}

Colloidal stability in aqueous medium is one of the most important characteristics associated with biomedical applications of nanoparticles $[28,29]$. Zeta potential measurement offers a key role in understanding the stability of nanoparticles [30]. From the Zeta potential diagrams presented in Fig. 3, it can be concluded that the stability of the citric acid coated IONPs presents a much improved stability compared to bare IONPs, since the Zeta potential absolute value is almost three times higher; i.e. $-26.6 \pm 3.57$ and $-9.51 \pm 3.8 \mathrm{mV}$, respectively [31].

\subsection{Atomic absorption spectroscopy analysis}

Iron (Fe) concentration of $\mathrm{Fe}_{3} \mathrm{O}_{4}$ nanoparticles coated with citric acid was determined AAS. The standard solution was analyzed and the absorbance was measured at the wavelength of $248.3 \mathrm{~nm}$ [32]. The standard curve of Fe concentration with absorbance at $248.3 \mathrm{~nm}$ was plotted in Fig. 4. Using the calibration curve, the concentration of $\mathrm{Fe}$ in $\mathrm{Fe}_{3} \mathrm{O}_{4}$ nanoparticles coated with citric acid was found to be $155.4 \mathrm{ppm}(0.1552 \mathrm{mg} / \mathrm{mL})$ 
which is safe and acceptable and does not present any toxicity threshold sensitive for medical applications including MRI [32].

\subsection{Magnetic resonance measurements}

Five samples of citric acid coated IONPs with different concentration of Fe were prepared to investigate their potential as contrast agent in MRI. To obtain $\mathrm{T}_{1}$ and $\mathrm{T}_{2}$ values, the images of agarose gel phantoms containing different solutions of Fe and blank sample were imported into image $\mathrm{J}$ software package and the signal intensities were measured. The images of the samples were obtained using spin-echo sequence device. For the relaxation time $T_{1}$, the values of the echo-time $\left(T_{E}\right)$ are fixed at $10 \mathrm{~ms}$ and that of the repetition time $\left(T_{R}\right)$ is varied as 100, 150, 200, 300, 600, 1200, 2400, 4800 and 8000 ms. For the relaxation time $T_{2}$, the values of $T_{R}$ are fixed at $1000 \mathrm{~ms}$ and that of $\mathrm{T}_{\mathrm{E}}$ is varied as 10, 15, 20, 30, 60, 120 and $200 \mathrm{~ms}$. The other parameters are kept fixed at field of view (FOV) $40 \mathrm{~mm}$ with an image matrix of 256x256 pixels, slice thickness 3 mm, number of cuts of 3 , and distance between slices is $0 \mathrm{~mm}$.

The relaxation times $T_{1}$ and $T_{2}$ for various Fe concentrations were obtained using single exponential fit intensity against $T_{R}$ and $T_{E}$. The values of $T_{1}$ for the samples with $0,0.125,0.25,0.5$, and $1.0 \mathrm{mg} / \mathrm{ml} \mathrm{Fe}$ are found to be $3157.66,2318.39,2015.49,1543.56$, and $1013.44 \mathrm{~ms}$, respectively. The relaxation time $\mathrm{T}_{1}$ decreases gradually and substantially ( 68\%) from 3157.66 to $1013.44 \mathrm{~ms}$ with the increase in the concentration of Fe from 0 to $1 \mathrm{mg} / \mathrm{ml}$. The results are summarized in Table 2 and plotted in Fig. 5.

Table 2

The signal intensities at fixed $T_{E}(10 \mathrm{~ms})$ and different $T_{R}(\mathrm{~ms})$ between 100 and $8000 \mathrm{~ms}$. The $T_{1}$ (ms) recovery time of each phantom with different concentration of IONPs coated with citric acid is obtained from curve fitting.

\begin{tabular}{|c|c|c|c|c|c|c|c|c|c|c|c|}
\hline & $\mathrm{TR}(\mathrm{ms})$ & 100 & 150 & 200 & 300 & 600 & 1200 & 2400 & 4800 & 8000 & $\mathrm{~T}_{1}(\mathrm{~ms})$ \\
\hline \multirow[t]{6}{*}{ Intensity } & Blank sample & 89 & 106 & 156 & 222 & 419 & 811 & 1360 & 1927 & 2331 & 3157.66 \\
\hline & (0 mg/ml) & & & & & & & & & & \\
\hline & $(0.125 \mathrm{mg} / \mathrm{ml})$ & 111 & 110 & 184 & 261 & 547 & 895 & 1430 & 1939 & 2172 & 2318.39 \\
\hline & $(0.25 \mathrm{mg} / \mathrm{ml}$ & 105 & 129 & 202 & 311 & 554 & 957 & 1535 & 1931 & 2135 & 2015.49 \\
\hline & $(0.5 \mathrm{mg} / \mathrm{ml})$ & 116 & 140 & 219 & 314 & 581 & 988 & 1384 & 1679 & 1795 & 1543.56 \\
\hline & (1 mg/ml) & 121 & 152 & 210 & 327 & 540 & 840 & 1067 & 1194 & 1214 & 1013.44 \\
\hline
\end{tabular}

Similarly, the values of $\mathrm{T}_{2}$ for the samples with $0,0.125,0.25,0.5$ and $1 \mathrm{mg} / \mathrm{ml} \mathrm{Fe}$ are found to be 173.61, 47.86, 32.40, 19.48 and $9.03 \mathrm{~ms}$, respectively. The relaxation time $\mathrm{T}_{2}$ decreases gradually and significantly ( 95\%) from 173.61 to $9.03 \mathrm{~ms}$ with the increase in the Fe concentration from 0 to $1 \mathrm{mg} / \mathrm{ml}$. The results are summarized in Table 3 and plotted in Fig. 6. Thus, for both $T_{1}$ and $T_{2}$-weighted images, it is observed that both $T_{1}$ and $T_{2}$ values decrease with the increase in Fe concentration, and this decrease is more significant for $T_{2}$. 
Table 3

The signal intensities at fixed $T_{R}(1000 \mathrm{~ms})$ and different $T_{E}$ between 10 and $200 \mathrm{~ms}$. The $\mathrm{T}_{2}$ (ms) recovery time of each phantom with different concentration of IONPs coated with citric acid is obtained from curve fitting.

\begin{tabular}{|llllllllll|}
\hline \multirow{2}{*}{ Intensity } & $\mathbf{1 0}$ & $\mathbf{1 5}$ & $\mathbf{2 0}$ & $\mathbf{3 0}$ & $\mathbf{6 0}$ & $\mathbf{1 2 0}$ & $\mathbf{2 0 0}$ & $\mathrm{T}_{2}$ (ms) \\
& $\begin{array}{l}\text { Blank sample } \\
(\mathbf{0 ~ m g} / \mathrm{ml})\end{array}$ & 703 & 679 & 642 & 582 & 494 & 358 & 250 & 173.61 \\
& & & & & & & & \\
\hline$(0.125 \mathrm{mg} / \mathrm{ml})$ & 785 & 677 & 645 & 502 & 268 & 81 & 43 & 47.86 \\
\hline$(0.25 \mathrm{mg} / \mathrm{ml}$ & 840 & 716 & 585 & 439 & 192 & 46 & 0 & 32.40 \\
\hline$(0.5 \mathrm{mg} / \mathrm{ml})$ & 838 & 632 & 480 & 305 & 79 & 0 & 0 & 19.48 \\
\hline$(1 \mathrm{mg} / \mathrm{ml})$ & 744 & 452 & 228 & 79 & 32 & 0 & 0 & 9.03 \\
\hline
\end{tabular}

The relaxation rates $\left(r_{1}\right.$ and $\left.r_{2}\right)$ are usually used to measure the enhancement efficiency of the contrast agent. To quantify the MRI contrast efficiency of citric acid coated IONPs, the $r_{1}$ and $r_{2}$ relaxation rates were obtained by measuring of relaxation times of different concentrations and plotting the inverse relaxation times (relaxation rates) versus different Fe concentrations, according to the following equation [33]:

$1 / T_{i, o b s}=1 / T_{i, \mathrm{~d}}+r_{i}[\mathrm{M}](\mathrm{i}=1,2)(2)$

where $1 / T_{i, \mathrm{obs}}$ denotes the measured solvent relaxation rate in the presence of a contrast agent, $1 / T_{i, \mathrm{~d}}$ is the solvent relaxation rate in the absence of a contrast agent, [M] represents the contrast agent concentration, and $r i$ is defined as the relaxation rate in $\mathrm{mM}^{-1} \cdot \mathrm{s}^{-1}$. The relaxation times $\left(1 / \mathrm{T}_{1}\right.$ and $\left.1 / \mathrm{T}_{2}\right)$ were plotted as a function of Fe concentration as shown in Fig. 8. As determined from the curve fitting in Fig. 8, the values of $r_{1}$ and $r_{2}$ of citric acid coated IONPs are found to be 0.654 and $103.02 \mathrm{mM}^{-1} . \mathrm{s}^{-1}$, respectively. The obtained results are due to the smaller distance between water molecules and magnetic core than that in the presence of nanoparticles. The smaller water molecules interact chemically with the magnetite particles' surface, and hence the water molecules cannot go close enough to the core as in the case of naked particles.

Reduced motion means that a higher proportion of $\mathrm{HOH}$ nuclei will experience both static fields (reducing $\mathrm{T}_{2}$ ) and interactions near the Larmor frequency (reducing $T_{1}$ ) [34]. The $r_{2} / r_{1}$ ratio is a key parameter to determine the efficiency of citric acid coated IONPs for MRI contrast agents [35]. Its value is found as 157.52, demonstrating that IONPs can be efficient as a $\mathrm{T}_{2}$ contrast agents.

Compared to other iron oxide-based contrast agents of similar sizes [36, 37], the values of $r_{2}$ of $103.02 \mathrm{mM}^{-1} . \mathrm{s}^{-}$ 1 and relaxivity ratio of 157.02 obtained in this study for IONPs coated with citric acid are found to be very high which is a clear indication for devising the same as an efficient $\mathrm{T}_{2}$ contrast agent.

The obtained results indicate that $r_{1}$ and $r_{2}$ values depend on surface modification (for instance coating with citric acid), the particles size of IONPs core, and the degree of aggregation [38]. The observed high $r_{2} / r_{1}$ ratios show that IONPs coated with citric acid can be considered as potential candidate to be utilized as MRI contrast 
agents in the future. We found that the particles synthesized in this work exhibit higher relaxivity ratios due to the presence of negative surface charges.

\section{Conclusion}

Pure and stable IONPs were produced using a cost-effective co-precipitation method with optimum properties obtained at $\mathrm{pH} 10$ which particle size about $11.91 \mathrm{~nm}$. Subsequent coating with citric acid as a capping agent has been performed in order to optimize surface functionalization and increase further the stability of IONPs. From the physicochemical analyses of the as-synthesized IONPs coated with citric acid by means of XRD, FTIR, AAS, and Zeta potential, the obtained results demonstrated that the method was successful for preparing high quality IONPs with a good colloidal stability. Due to the high magnetic moment of the as-synthesized IONPS coated with citric acid, the sensitivity of the composite particles as MRI contrast agent was evaluated. The results revealed that citric acid coated IONPs rapidly shortens $T_{2}$ and $T_{1}$ relaxation times of tissue equivalent Agarose phantom by $94.7 \%$ and $67.9 \%$, respectively. Moreover, the efficiency evaluated from the relaxivity ratio indicated IONPs coated with citric acid were highly effective for MRI T 2 contrast agent with $r_{2} / r_{1}$ value of 157.52. Finally, it was concluded that the sensitivity of IONPs coated with citric acid prepared demonstrate a potential as a candidate for MRI contrast agent.

\section{Declarations}

\section{Acknowledgment}

Authors would like to thank the Deanship of Research at Jordan University of Science and Technology for supporting and funding this research project (Grant \# 55/2020). The author also would like to thank Prince Sultan University for their support.

\section{Funding}

"The authors declare that no funds, grants, or other support were received during the preparation of this manuscript."

\section{Competing Interests}

"The authors have no relevant financial or non-financial interests to disclose."

\section{Author Contributions}

"Authors contributed equally to the manuscript and all authors commented on previous versions of the manuscript. All authors read and approved the final manuscript."

\section{Data Availability}

"The datasets generated during and/or analysed during the current study are available from the corresponding author on reasonable request."

\section{References}


1. O.A. Noqta, A.A. Aziz, I.A. Usman, M. Bououdina, Recent advances in iron oxide nanoparticles (IONPs): synthesis and surface modification for biomedical applications. J. Supercond. Novel Magn. 32(4), 779795 (2019)

2. M.A. Dheyab, A.A. Aziz, M.S. Jameel, O.A. Noqta, B. Mehrdel, Synthesis and coating methods of biocompatible iron oxide/gold nanoparticle and nanocomposite for biomedical applications. Chin. J. Phys. 64, 305-325 (2020)

3. J. Gao, H. Gu, B. Xu, Multifunctional Magnetic Nanoparticles: Design, Synthesis, and Biomedical Applications. Acc. Chem. Res. 42, 1097-1107 (2009)

4. I.M. Hsing, Y. Xu, W. Zhao, Micro- and Nano- Magnetic Particles for Applications in Biosensing. Electroanalysis 19, 755-768 (2007)

5. T.C.L. Ankamwar, J.H. Huang, R.S. Liu, M. Hsiao, C.H. Chen, Y.K. Hwu, Biocompatibility of Fe304 Nanoparticles Evaluated by In Vitro Cytotoxicity Assays Using Normal, Glia And Breast Cancer Cells. Nanotechnology 21, 1-9 (2010)

6. T. Hyeon, L.S. Park, J. Chung Y \& H.B. Na, Synthesis of Highly Crystalline and Monodisperse Maghemite Nanocrystallites without a Size Selection Process. J. Am. Chem. Soc. 123, 12798-12128 (2001)

7. B. Behdadfar, K.A. Sadeghi-Aliabadi, H. Morales MDP \& M. Mozaffari, Synthesis of High Intrinsic Loss Power Aqueous Ferrofluids of Iron Oxide Nanoparticles by Citric Acid-Assisted Hydrothermal-Reduction Route. J. Solid State Chem. 187, 2026 (2011)

8. A. Bumb, B.M. Choyke, P.L. Fugger, L. Eggeman, A. Prabhakaran, D. Hutchinson J \& P.J. Dobson, Synthesis and Characterization of Ultra-Small Superparamagnetic Iron Oxide Nanoparticles Thinly Coated with Silica. Nanotechnology 19, 1-6 (2008)

9. H. Nosrati, M. Salehiabar, H.K. Manjili, H. Danafar, S. Davaran, Preparation of magnetic albumin nanoparticles via a simple and one-pot desolvation and co-precipitation method for medical and pharmaceutical applications. Int. J. Biol. Macromol. 108, 909-915 (2018)

10. R. Qiao, C. Yang, M. Gao, Superparamagnetic iron oxide nanoparticles: from preparations to in vivo MRI applications. J. Mater. Chem. 19(35), 6274-6293 (2009)

11. E.M. Materón, C.M. Miyazaki, O. Carr, N. Joshi, P.H. Picciani, C.J. Dalmaschio, F. Davis, F.M. Shimizu, Magnetic nanoparticles in biomedical applications: A review. Appl. Surf. Sci. Adv. 6, 100163 (2021)

12. N. Mohamad Nor et al., Properties of surface functionalized iron oxide nanoparticles (ferrofluid) conjugated antibody for lateral flow immunoassay application. J. Alloys Compd. 538(0), 100-106 (2012)

13. ÂL. Andrade et al., Effect of tetramethylammonium hydroxide on nucleation, surface modification and growth of magnetic nanoparticles. Journal of Nanomaterials, 2012. 2012: p. 15.

14. J.-F. Lutz et al., One-Pot Synthesis of PEGylated Ultrasmall Iron-Oxide Nanoparticles and Their in Vivo Evaluation as Magnetic Resonance Imaging Contrast Agents. Biomacromolecules 7(11), 3132-3138. (2006)

15. C. Blanco-Andujar et al., Elucidating the morphological and structural evolution of iron oxide nanoparticles formed by sodium carbonate in aqueous medium. J. Mater. Chem. 22(25), 12498-12506 (2012)

16. L. Vayssieres et al., Size tailoring of magnetite particles formed by aqueous precipitation: An example of thermodynamic stability of nanometric oxide particles. J. Colloid Interface Sci. 205(2), 205-212. (1998) 
17. S.-J. Lee et al., Synthesis and characterization of superparamagnetic maghemite nanoparticles prepared by co-precipitation technique. J. Magn. Magn. Mater. 282(0), 147-150 (2004)

18. G. Gnanaprakash et al., Effect of Digestion Time and Alkali Addition Rate on Physical Properties of Magnetite Nanoparticles. J. Phys. Chem. B 111(28), 7978-7986. (2007)

19. M. Niederberger, Nonaqueous sol-gel routes to metal oxide nanoparticles. Acc. Chem. Res. 40(9), 793800. (2007)

20. R.J.P.a.D.L. Corriu, Recent Developments of Molecular Chemistry for Sol-Gel Processes. Angewandte Chemie International Edition in English 35(1314), 1420-1436 (1996)

21. A. Avasthi et al., Magnetic Nanoparticles as MRI Contrast Agents. Top. Curr. Chem. 378(3), 40 (2020)

22. K.-S.L. Loh, Y. Musa, A. Salmah, A.A. Zamri, I. Use of Fe304 Nanoparticles for Enhancement of Biosensor Response to the Herbicide 2,4-Dichlorophenoxyacetic Acid. Sensors 8, 5775-5791 (2008)

23. M. Imran, N. Zouli, T. Ahamad, S.M. Alshehri, M.R. Chandan, S. Hussain, A. Aziz, M.A. Dar, A. Khan, Carboncoated Fe 304 core-shell super-paramagnetic nanoparticle-based ferrofluid for heat transfer applications. Nanoscale Advances, 2021. 3(7): p. 1962-1975

24. L. Bondarenko, V. Terekhova, A. Kahru, G. Dzhardimalieva, E. Kelbysheva, N. Tropskaya, K. Kydralieva, Sample preparation considerations for surface and crystalline properties and ecotoxicity of bare and silicacoated magnetite nanoparticles. RSC Adv. 11(51), 32227-32235 (2021)

25. S. Devesa, A. Rooney, M. Graça, D. Cooper, L. Costa, Williamson-hall analysis in estimation of crystallite size and lattice strain in Bi1. 34FeO. 66Nb1. 3406. 35 prepared by the sol-gel method. Materials Science and Engineering: B, 2021. 263: p. 114830

26. M.H.R. Farimani, N. Shahtahmasebi, M.R. Roknabadi, N. Ghows, A. Kazemi, Study of structural and magnetic properties of superparamagnetic $\mathrm{Fe} 3 \mathrm{O} 4 / \mathrm{SiO} 2$ core-shell nanocomposites synthesized with hydrophilic citrate-modified Fe304 seeds via a sol-gel approach. Phys. E: Low-dimensional Syst. Nanostruct. 53, 207-216 (2013)

27. U. Patel, K. Chauhan, S. Gupte, Synthesis, characterization and application of lipase-conjugated citric acidcoated magnetic nanoparticles for ester synthesis using waste frying oil. 3 Biotech. 8(4), 1-12 (2018)

28. O.A. Noqta, B.K. Sodipo, A.A. Aziz, One-pot synthesis of highly magnetic and stable citrate coated superparamagnetic iron oxide nanoparticles by modified coprecipitation method. Funct. Compos. Struct. 2(4), 045005 (2020)

29. O. Abu-Noqta, A. Aziz, A. Usman, Colloidal stability of Iron oxide nanoparticles coated with different capping agents. Materials Today: Proceedings, 2019. 17: p. 1072-1077

30. A. Rawle, Basic of principles of particle-size analysis. Surface coatings international. Part A, 86 (C.j, 2003), pp. 58-65

31. L. Li, K. Mak, C.W. Leung, K. Chan, W. Chan, W. Zhong, P. Pong, Effect of synthesis conditions on the properties of citric-acid coated iron oxide nanoparticles. Microelectron. Eng. 110, 329-334 (2013)

32. B. Gómez-Nieto, M.J. Gismera, M.T. Sevilla, J.R. Procopio, Simultaneous and direct determination of iron and nickel in biological solid samples by high-resolution continuum source graphite furnace atomic absorption spectrometry. Talanta 116, 860-865 (2013)

33. P.M. Conn, Imaging in Biological Research, Part B (Elsevier, 2004) 
34. B.H. Kim, L.N. Kim, H. An, K. Park, Y.I. Choi, Y, et al. Large-Scale Synthesis of Uniform and Extremely SmallSized Iron Oxide Nanoparticles for High-Resolution T1 Magnetic Resonance Imaging Contrast Agents. J. Am. Chem. Soc. 133(32), 12624-12631 (2011)

35. T. Passuello, P.M. Piccinelli, F. Polizzi, S. Marzola, P. Tambalo, S, et al. PEGcapped, lanthanide doped GdF3 nanoparticles: luminescent and T2 contrast agents for optical and MRI multimodal imaging. Nanoscale 4(24), 7682-7689 (2012)

36. A. Saraswathy, N.S. Jeevan, M. Nimi, N. Arumugam, S. Harikrishnan, VS, et al. Citrate coated iron oxide nanoparticles with enhanced relaxivity for in vivo magnetic resonance imaging of liver fibrosis. Colloids Surf., B 117, 216-224 (2014)

37. J. Pellico, R.-C.J. Fernández-Barahona, I. Gutiérrez, L. Lechuga-Vieco, A.V. Enríquez, JA, et al. One-Step Fast Synthesis of Nanoparticles for MRI: Coating Chemistry as the Key Variable Determining Positive or Negative Contrast. Langmuir 33(39), 10239-10247 (2017)

38. Y.-J. Jang, S. Liu, H. Yue, J. Park, H. Cha, S.L. Ho, S. Marasini, A. Ghazanfari, M.Y. Ahmad, X. Miao, Hydrophilic biocompatible poly (acrylic acid-co-maleic acid) polymer as a surface-coating ligand of ultrasmall Gd203 nanoparticles to obtain a high r1 value and T1 MR images. Diagnostics 11(1), 2 (2021)

\section{Figures}

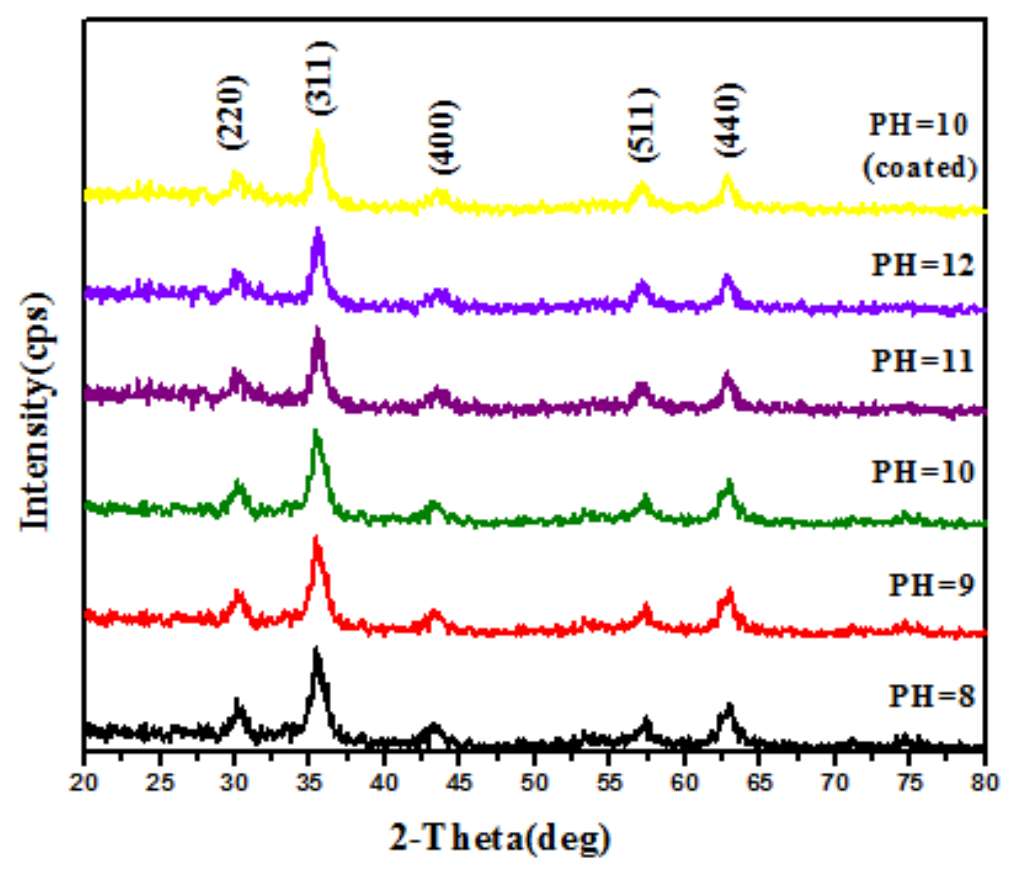

Figure 1

X-ray diffraction patterns of $\mathrm{Fe}_{3} \mathrm{O}_{4}$ nanoparticles at different $\mathrm{pH}$ values. 


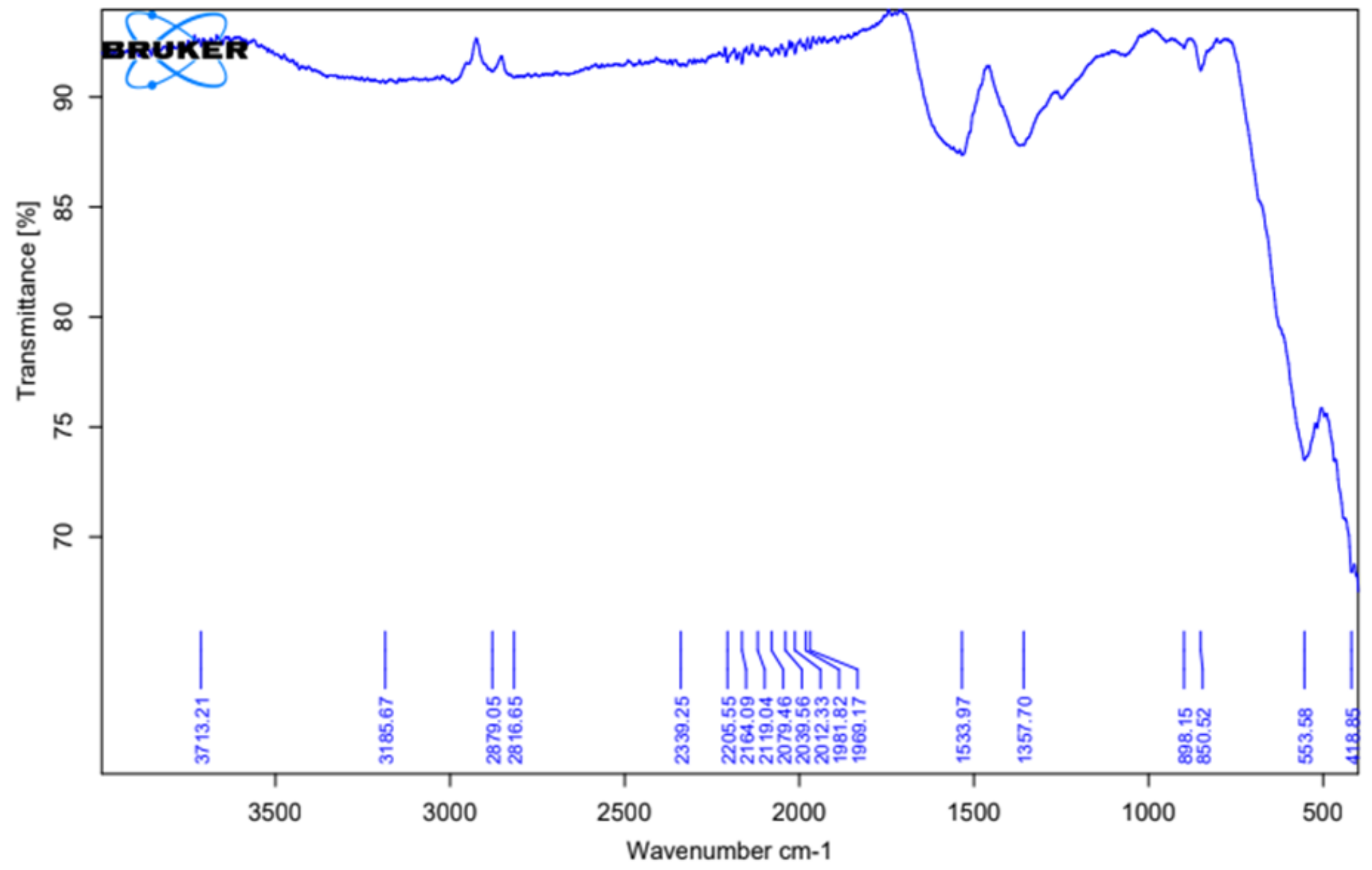

Figure 2

FTIR Spectrum of $\mathrm{Fe}_{3} \mathrm{O}_{4}$ nanoparticles prepared at pH 10 and coated with citric acid. 

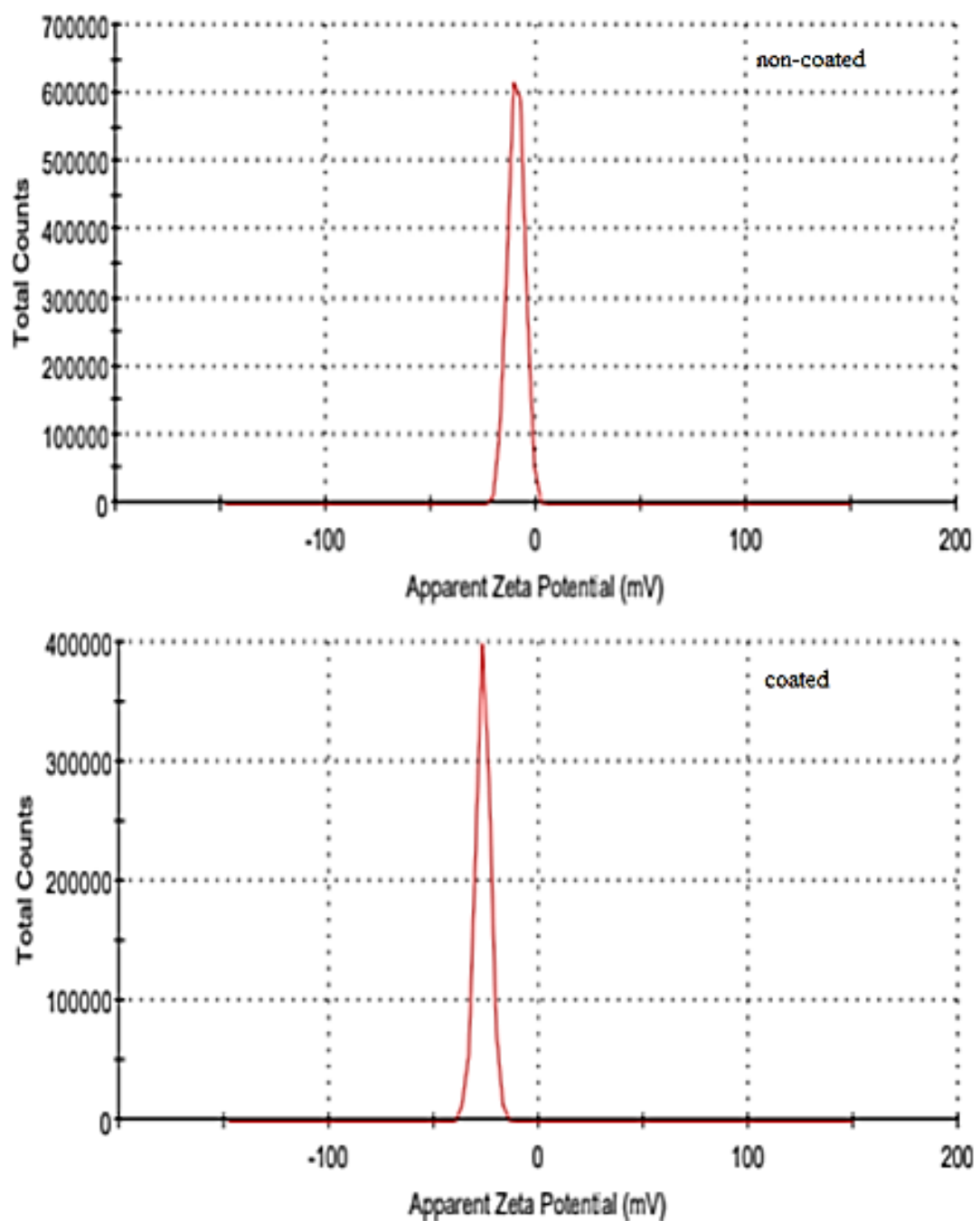

Figure 3

Zeta Potential distribution for the coated and non-coated $\mathrm{Fe}_{3} \mathrm{O}_{4}$ nanoparticles prepared at pH 10. 


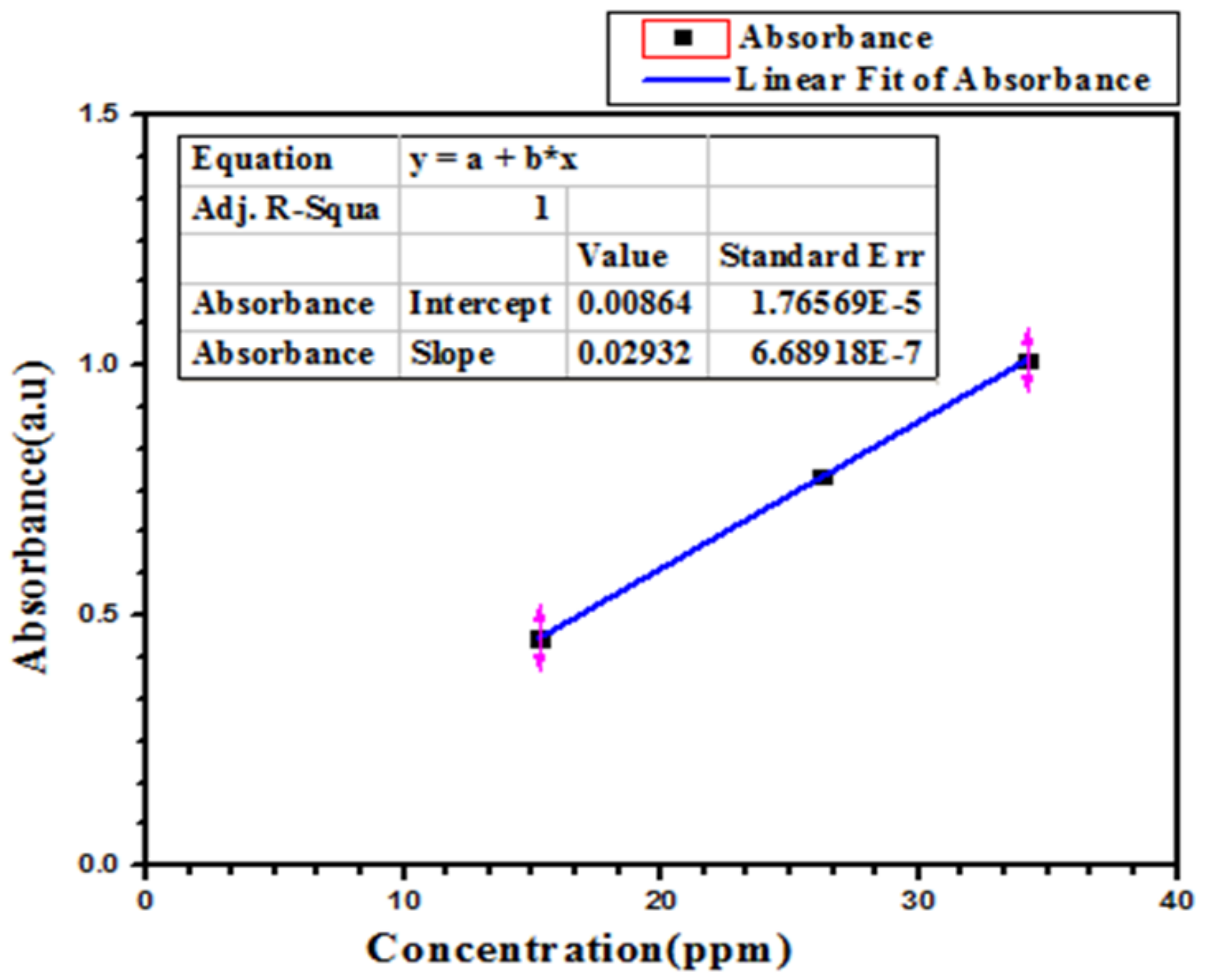

Figure 4

Atomic absorption spectrum $\mathrm{Fe}_{3} \mathrm{O}_{4}$ nanoparticles prepared at $\mathrm{pH} 10$ and coated with citric acid. 


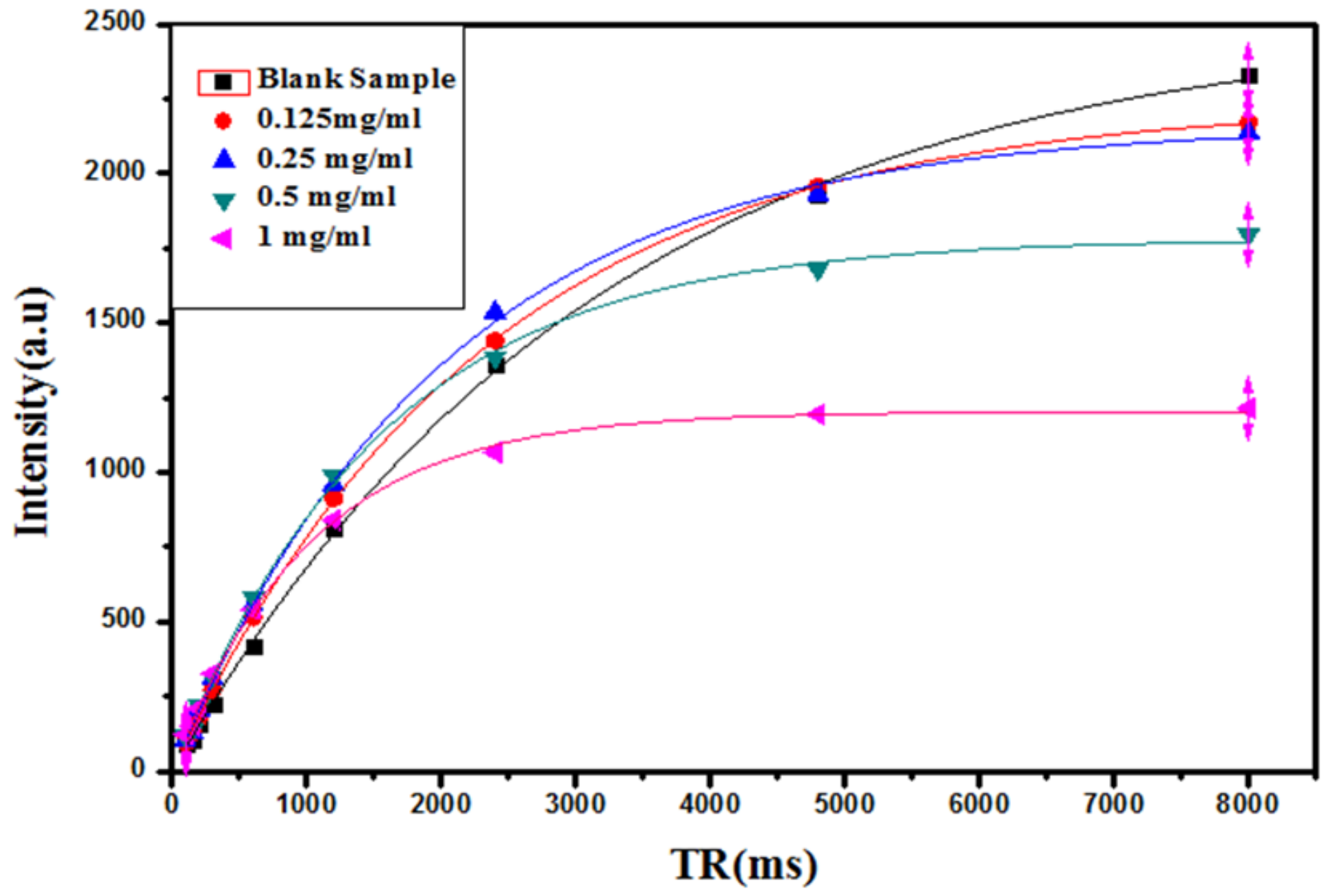

Figure 5

The intensity as a function of echo time $T_{R}$ for different Fe concentrations. 


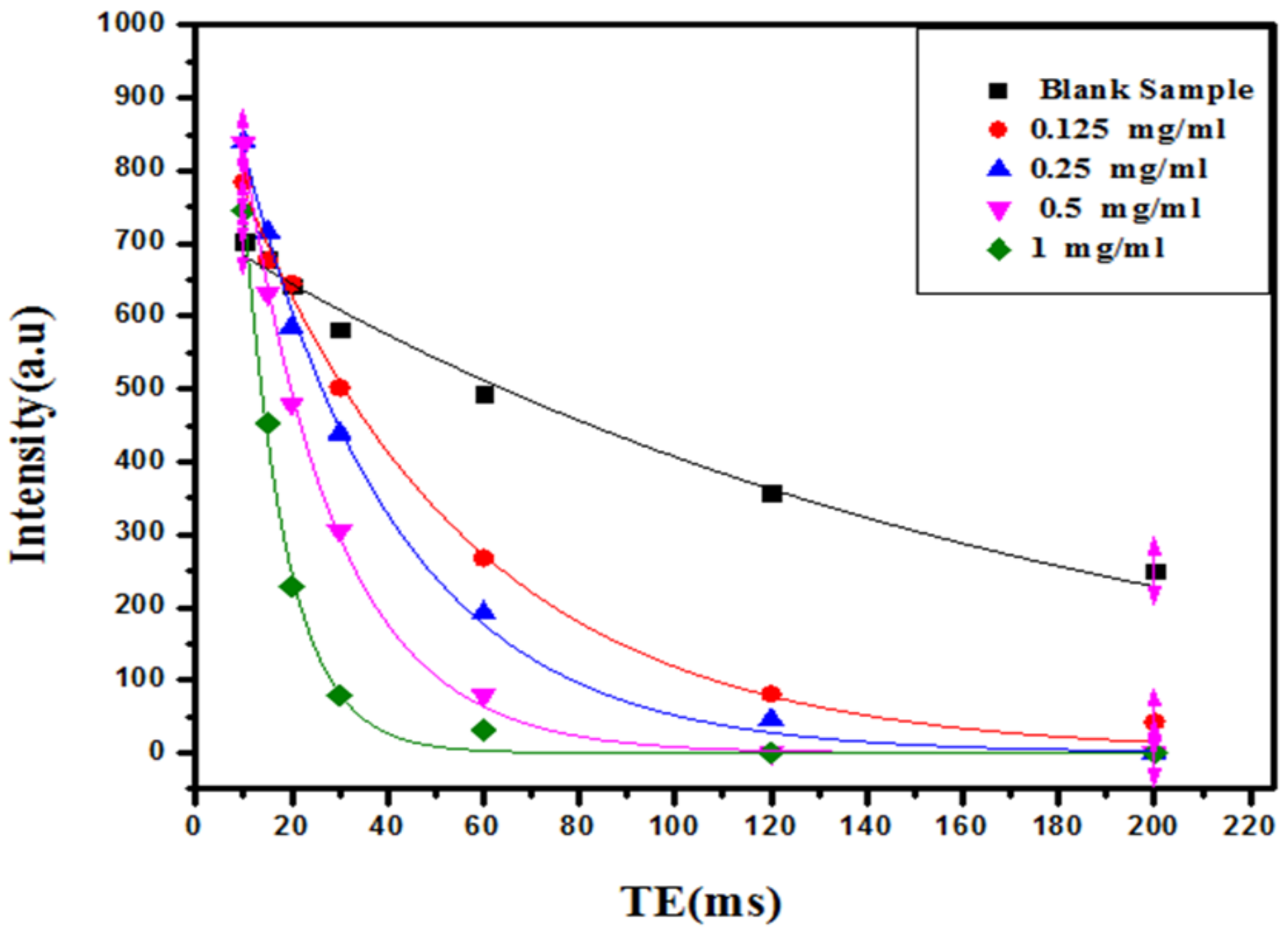

Figure 6

The intensity as a function of repetition time $T_{E}$ for different Fe concentrations. 
(a)

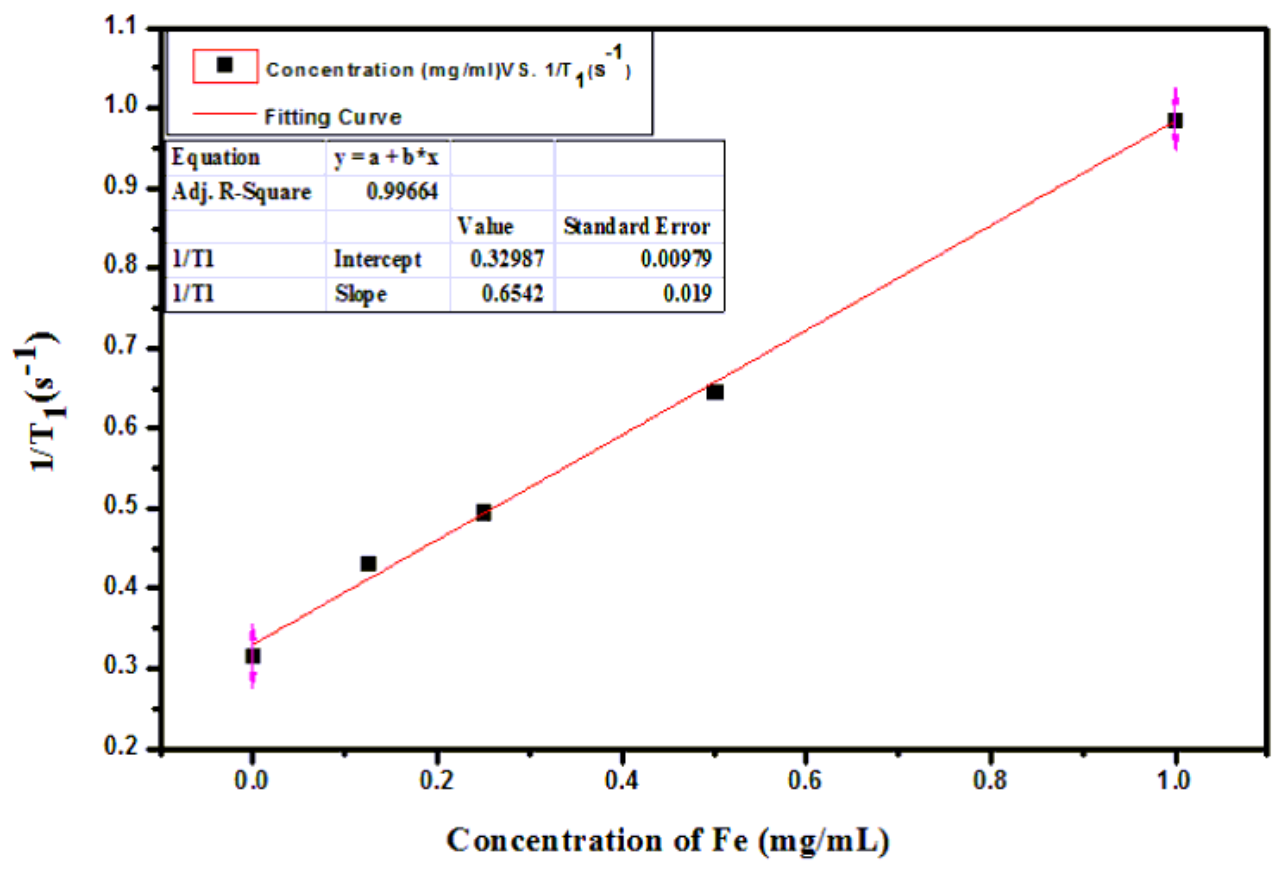

(b)

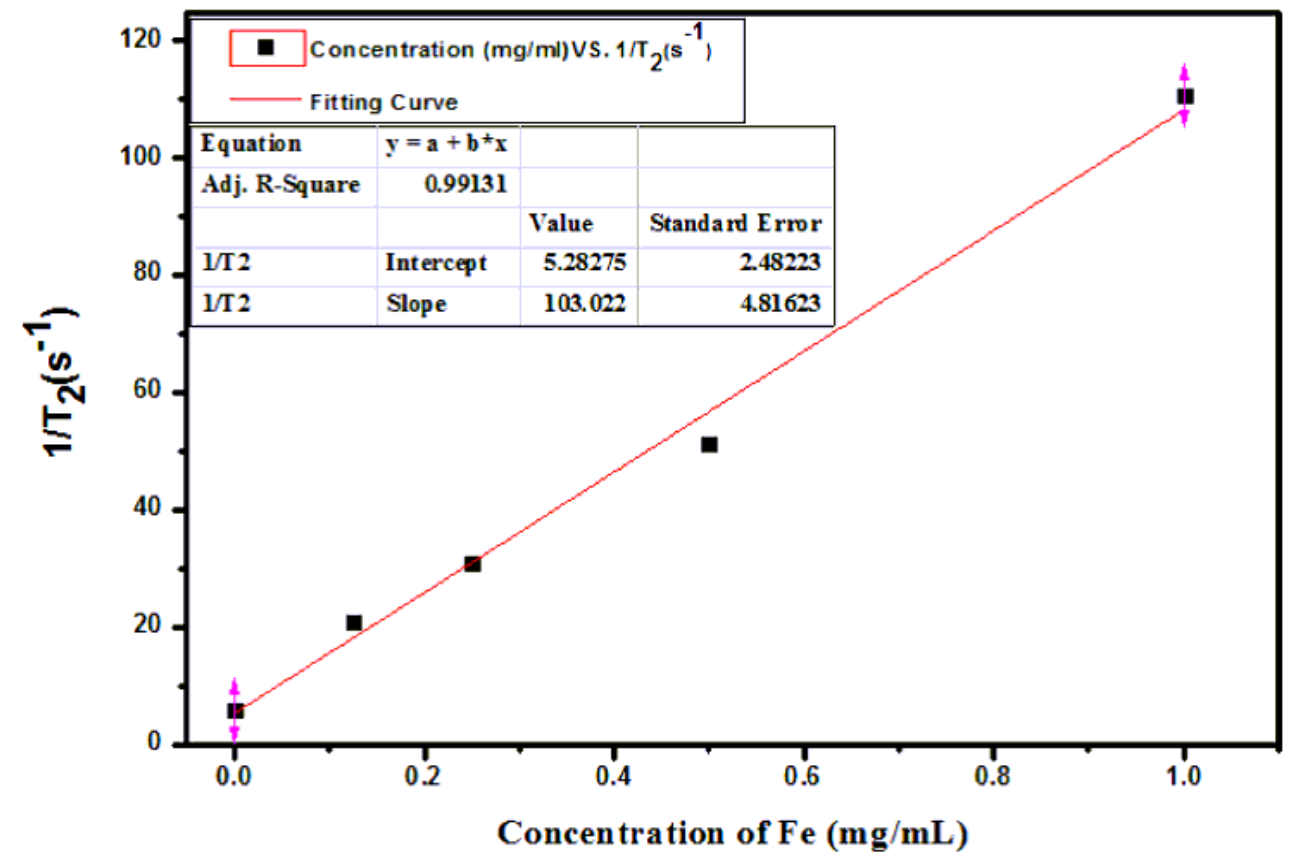

Figure 7

The relaxivity graph of (a) $1 / T_{1}$ and (b) $1 / T_{2}$ against different Fe concentrations. 